\title{
How Lac Repressor binds to Lac DNA
}

THE experiments published in Nature two weeks ago (Nature, 237, 323 ; 1972) by Müller-Hill and his several colleagues, which have led to a structural model of how lac repressor protein binds specifically to the operator region of the lac operon, provide a remarkable example of the sophistication that is nowadays the hallmark of the molecular geneticist.

Ever since Gilbert and Müller-Hill first isolated lac repressor protein in 1967, evidence has been steadily accumulated, all of which indicates that the lac repressor protein prevents the coordinated expression of the three structural genes of the lac operon by binding to a specific region of the operon called the operator. And it is envisaged that in so doing the repressor blocks DNA dependent RNA polymerase from binding to the operon and transcribing it into a single messenger RNA molecule containing the information to specify the three enzymes of the operon. Furthermore, investigations of pure repressor protein, which of course can also bind inducers of the operon and as a result suffer an allosteric change which prevents its binding to the operator, have revealed that, with a molecular weight of about 160,000 , it is made of four identical polypeptide subunits and that it does not contain nucleic acid.

All these observations provide more than a superficial description of the natural history of the lac repressor but they do not satisfy anybody curious about the structure of the repressor, the topography of its interaction with the operator and the basis of the specificity of this interaction.

From the many precedents provided by enzymologists who have shown that the active sites of enzymes are usually located in clefts in enzyme molecules into which substrate molecules can fit, it seemed reasonable to anticipate that the lac operator DNA would be found to fit in a cleft in the repressor molecule. The data belie any such expectations, however, for Müller-Hill and his associates believe on the contrary that protrusions from the repressor molecule, comprising the first (5') fifty amino-acids of each subunit, specifically bind to the operator region.

Genetic data provided the first clue that the $5^{\prime}$ terminal regions of the repressor subunits are involved in the specific binding to DNA. Mutants of the lac repressor which have lost the ability to bind to the operator but can still bind inducer molecules all map at the $5^{\prime}$ end of the repressor gene which specifies the first fifty aminoacids of the repressor protein. Moreover, genetic analyses indicate that the operator and inducer binding sites are not very closely connected.

Analysis of the first fifty aminoacids at the $5^{\prime}$ terminus of the repressor subunit chain revealed only one unusual feature, this short region of the molecule contains half the total tyrosine residues, but the sequence is otherwise unremarkable. Some 247 point mutations, however, which result in the change of one or another of these fifty amino-acids all also result in a repressor which cannot bind to the operator DNA. This region of the molecule is most probably therefore directly involved in this binding. A sequence of fifty amino-acids is, however, scarce enough to form a cleft, but it could form a protrusion from the repressor molecule and if the protruding stretch assumed an alpha helical configuration it could fit into the deeper of the two grooves of $B$ form double helical DNA.

By playing, as they put it, with space filling molecular models, the Cologne group found that only one part of the $5^{\prime}$ terminal region of a repressor subunit-that between amino-acids 17 and 33-can fit into the deeper groove of double stranded DNA and simultaneously bind both to phosphates of the DNA backbone and the polar groups of the bases in the groove in such a way that the four possible base pairs can be distinguished from each other. This segment of the repressor subunit, they argue, is therefore critical for binding to the operator DNA. But that is not all; from the space filling models Müller-Hill and his colleagues have predicted the sequence of eight base pairs which are recognized by the protruding $5^{\prime}$ end of a repressor subunit and they believe this sequence may, in the operator region, be repeated four times, with intervening spacers, so that each subunit of the repressor can recognize the same DNA sequence.

Of course it would be surprising indeed if in every detail this model proves to be correct, but at the very least it should greatly stimulate attempts to sequence the operator DNA and once that sequence is known the validity or otherwise of the model will be immediately apparent. -From a Correspondent.

\section{Feline Leukaemia Virus sensitive to Interferon}

IN next Wednesday's Nature New Biology (June 28) Merigan, Rodgers, Hardy, Old and Kassel add feline leukaemia virus (FeLV) to the list of RNA tumour viruses that have been shown to be sensitive to interferon. Merigan and his colleagues prepared feline interferon from the feline kidney cell line CrFK which they had infected with Newcastle disease virus. They then assayed the ability of this interferon to block the infection of CrFK cells and cells of the feline fibroblast line, FFc60wf, by FeLV. The virus was prepared by membrane filtration of supernatant fluid from cultures of cat cells chronically infected with FeLV, and infection of the CrFK and FFc60wf cells was assayed by an indirect immunofiuorescent antibody test which detects in cells the FeLV groupspecific antigen.

By treating cells with the interferon 24 hours before their infection with FeLV, Merigan et al. caused a signifi" cant decrease in the number of infected cells. As in other systems the dose response curve of the interferon proved to be sigmoidal and the maximum inhibition of infection was some 80 per cent, which, as Merigan et al. comment, may mean that a minority of FeLV may be resistant to the action of interferon.

The results of a range of tests, including parallel assays of the antivesicular stomatitis virus activity of the interferon and of the effects of varying the time and duration of exposure of the cells to interferon, indicate conclusively that it is the interferon itself and not some contaminant of the interferon preparations which is aborting the FeLV infection. Moreover, exposure of the cat cells to interferon alone proved that, at least at the concentrations used, it is not cytotoxic.

Like Rous sarcoma virus and murine sarcoma and leukaemia viruses, feline leukaemia virus is apparently sensitive to interferon in vitro, and now Merigan et al. are investigating the effects of interferon on cats with virus associated leukaemia. 